\title{
Structure and Mixing Properties of Pressure-Atomized Sprays
}

\author{
G. A. Ruff," A. D. Sagar, $\uparrow$ and G. M. Faeth $\ddagger$ \\ University of Michigan, Ann Arbor, Michigan
}

\begin{abstract}
A theoretical and experimental study of the dense-spray region of pressure-atomized nonevaporating sprays is described, emphasizing flows in the wind-induced and atomization breakup regimes. Mean and fluctuating velocities at the injector exit, mean liquid volume fraction distributions, and entrainment rates were measured for large-scale (9.5 and $19.1 \mathrm{~mm}$ injector diameters) water jets in still air at atmospheric pressure. It was found that mixing was strongly influenced by the degree of flow development at the injector exit and the breakup regime: fully developed injector flow and atomization breakup yielded the fastest mixing rates. Predictions based on the locally homogeneous flow approximation, where relative velocities between the phases were neglected, gave encouraging predictions of dense-spray properties in the near-injector region for atomization breakup, including representation of flow development effects at the injector exit.
\end{abstract}

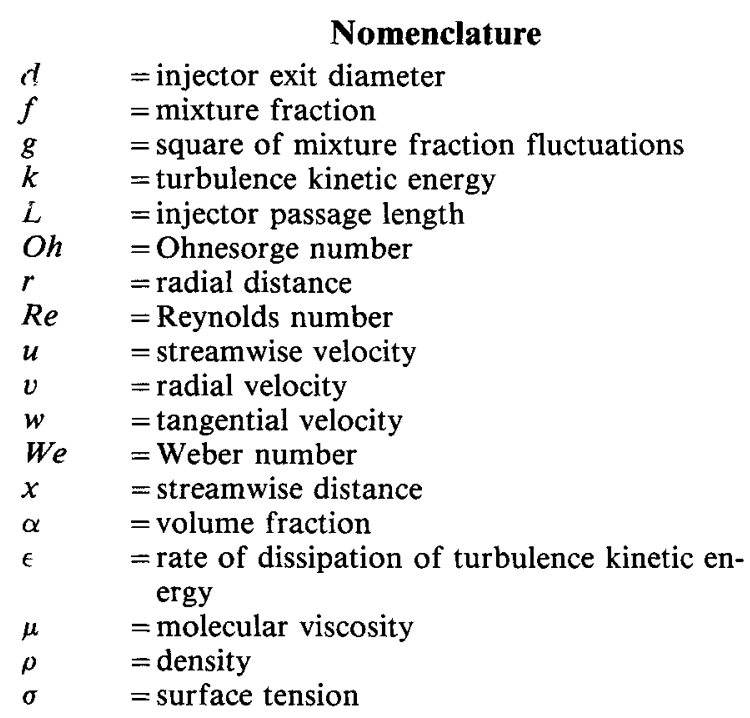

Subscripts

$c \quad=$ centerline value

$f \quad=$ liquid-phase property

$g \quad=$ gas-phase property

$3=$ injector exit condition

Superscripts

$\left(^{-}\right),\left(^{-}\right)^{\prime}=$ time-averaged mean and rms fluctuating quantities

$\left(^{\sim}\right),\left(^{\sim}\right)=$ Favre-averaged mean and rms fluctuating quantities

\section{Introduction}

$\mathbf{L}$ IQUID injection into a gas is an important fundamental flow, since it is the multiphase counterpart of the single-phase jet. This flow also has practical applications for pressure-atomized sprays, which are common in propulsion and power systems; e.g., pressure atomization is used for fwel or propellant injectors of afterburners, liquid rocket en-

Received Nov. 18, 1987; revision received June 15, 1988. Copyright (C) 1988 American Institute of Aeronautics and Astronautics, Inc. All rights reserved.

${ }^{*}$ Graduate Assistant, Department of Aerospace Engineering.

†Graduate Assistant, Department of Aerospace Engineering; currently with Materials Science and Engineering Department, Massachusetts Institute of Technology, Cambridge, MA.

$\ddagger$ Professor, Department of Aerospace Engineering. Fellow AIAA. gines, and fuel-injected internal combustion engines. Motivated by these observations, the present investigation considered the dense-spray region of nonevaporating pressure-atomized sprays in a still environment. Spray structure was measured in the near-injector region to gain a better understanding of breakup and mixing properties of the flow. The new measurements were also used to evaluate analysis of the flow, based on the locally homogeneous flow (LHF) approximation of multiphase flow theory, i.e., assuming that interphase momentum transport rates are infinitely fast, so that both phases have the same instantaneous velocity and are in thermodynamic equilibrium at each point in the flow.

The properties of pressure-atomization sprays are strongly influenced by their breakup regime. As injector flow rates increase, the flow passes through a succession of breakup regimes, as follows ${ }^{1,2}$ : drip, Rayleigh, first wind-induced, second wind-induced, and atomization. Drip breakup involves interactions between surface tension and gravity since fluid inertia is ineligible in this regime: large drops are formed at the injector exit that then fall as a single stream. Rayleigh breakup involves interactions between fluid inertia and surface tension: drops having diameters greater than the injector diameter are formed at some distance from the injector. Wind-induced breakup involves instabilities caused by the relative motion of the gas and the liquid, stabilized by surface tension. First wind-induced breakup is caused by instability of the whole liquid column: drops having diameters comparable to the injector diameter are formed far from the injector. Second windinduced breakup involves surface instabilities of the liquid column: drops having diameters much smaller than the injector diameter are formed, beginning at various distances from the injector. The point of breakup moves progressively toward the injector exit as jet velocities increase in the second windinduced breakup regime. The atomization breakup regime begins when the point of breakup reaches the injector exit and persists for all higher injector velocities. The present study was limited to wind-induced and atomization breakup, since these regions are most important for practical applications, e.g., the other regimes are limited to a narrow range of low injector flow rates.

A pressure-atomization spray has several flow regions during atomization breakup, as follows: a single-phase liquid flow in the injector passage, a dilute-spray region involving roughly spherical drops with liquid volume fraction less than $1-10 \%$ at the periphery of the spray and far from the injector, and a dense-spray region in the core of the flow near the injector exit. The dense-spray region contains a contiguous allliquid core, similar to the potential core of a single-phase jet, that is surrounded by a shear layer containing drops, ligaments, and other irregularly shaped liquid elements. Aside 
from these general features, however, the properties of the dense-spray region are not well known because of problems of flow visualization and the limited reliability of probe measurements when liquid volume fractions are high. Even past measurements of the length of the all-liquid core are widely scattered and controversial. ${ }^{3-5}$

Separated-flow analysis has proved to be effective and is under active development for dilute sprays. ${ }^{6,7}$ Since dense sprays involve liquid elements having complex and unknown shapes and strong interactions between liquid elements, separated-flow analysis is hard to formulate precisely and has not attracted much attention for analyzing these flows. Thus, LHF analysis of dense sprays has been studied as an alternative, although its effectiveness is controversial. Wu et al. ${ }^{8,9}$ report measurements of spray angles and drop velocities in nonevaporating pressure-atomized sprays at elevated pressures, concluding that use of the LHF approximation is appropriate for these conditions. On the other hand, Mao et al. ${ }^{10}$ found that LHF analysis of combusting pressureatomized sprays at high pressures gave useful qualitative information, but had only limited quantitative accuracy due to significant drop inertia in the rapidly decelerating flowfield of typical sprays. Experimental evidence on both sides of the controversy, however, came from regions where the spray was actually dilute and is not very convincing. This further complicates the issue, since detailed measurements of the structure of dilute sprays generally show the LHF analysis overestimates rates of flow development and is less effective than separatedflow methods. ${ }^{11-14}$

The present investigation was undertaken to help resolve these controversies concerning the structure of the dense-spray region of pressure-atomized sprays and the effectiveness of the LHF approximation of estimating the properties of this flow. Measurements of mean liquid volume fractions using gammaray absorption and entrainment using laser Doppler anemometry (LDA) were made to provide information concerning the structure and mixing properties of the dense-spray region. Both techniques avoid problems of obscuration of optical diagnostics and uncertainties of probe measurements at high liquid volume fractions. Predictions of spray structure with existing methods of LHF analysis ${ }^{7}$ were also evaluated using the new measurements.

The paper begins with a description of experimental and theoretical methods. Flow visualization is then used to identify breakup regimes and physical phenomena of interest. The paper concludes with discussion of the liquid volume fraction and entrainment measurements and their comparison with predictions based on the LHF approximation. The following description is brief; more details are reported elsewhere. ${ }^{15}$

\section{Experimental Methods}

\section{Apparatus}

Issues being studied relate to the dynamics of turbulent spray mixing processes, which are not thought to be strongly influenced by the injector diameter; therefore, large-scale $\mathbf{( 9 . 5}$ and $19.1 \mathrm{~mm}$ diam) injector passages were used to get adequate spatial resolution for the measurements. Water was used as the test liquid, injected vertically downward in still air at normal temperature and pressure. The water was collected in a baffled tub to prevent splashing up into the area where measurements were made and then discharged to a drain. City water was supplied to the injector using a centrifugal pump. The rate of water flow was adusted using a bypass system and measured using a turbine flow meter, which was calibrated by collecting water for timed intervals.

Three injectors were used: a slug flow injector having an exit diamter of $9.5 \mathrm{~mm}$ and two fully developed flow injectors having diameters of 9.5 and $19.1 \mathrm{~mm}$. The slug flow injector consisted of a honeycomb flow straightener $(1.6 \mathrm{~mm}$ cells, 25 $\mathrm{mm}$ long) and two screens to calm the flow $(16 \times 16$ square mesh, $0.18 \mathrm{~mm}$ diam wire) followed by a 13.6:1 area contraction to the injector exit. The contraction followed the contour prescribed by Smith and Wang, ${ }^{16}$ to obtain a uniform (slug) flow having low turbulence intensities at the exit. The fully developed flow injectors used the same flow straightener followed by constant-diameter passages having lengths of 41 passage diameters. Instrumentation was mounted rigidly; therefore, flow structure was measured by traversing the injectors horizontally (up to $1 \mathrm{~m}$, with a positioning accuracy of 5 $\mu \mathrm{m}$ ) and vertically (up to $2 \mathrm{~m}$, with a positioning accuracy of $0.5 \mathrm{~mm}$ ).

\section{Instrumentation}

\section{Flow Visualization}

Flash photography was used to study the appearance of the sprays, based on a Xenon Corp., high-intensity micropulse system (model $457 \mathrm{~A}$ ), which provided a $10 \mathrm{~J}$ light pulse with a $1 \mu$ s duration. The photographs were obtained in a darkened room with the flash lamp controlling the time of exposure, us ing a $4 \times 5$ Speed Graphic camera loaded with Polaroid, type 57 black-and-white film (ASA 3000). The camera was directed normal to the spray axis from a position near the flash lamp. The sprays were photographed in $250 \mathrm{~mm}$ long sections in order to provide reasonable spatial resolution.

\section{Gamma-Ray Absorption}

Distributions of mean liquid volume fractions were measured using gamma-ray absorption. An iodine 125 isotope source ( $2 \mathrm{mCi}$, emitting primarily at $27.47 \mathrm{keV}$ ) provided a soft gamma-ray source that had good absorption levels in order to minimize experimental uncertainties. The source was placed in a lead casket having a collimating aperture with a diameter of $1.6 \mathrm{~mm}$ and a length of $13 \mathrm{~mm}$. Gamma rays passing through the flow were detected and counted with a Bicron $x-$ ray probe (model $1 \times$ M.040/1.54) and an EG\&G Ortec singlechannel analyzer and counter/timer (models 556, 590A, 974). A lead aperture (1.5-6 $\mathrm{mm}$ in diameter, depending on position, and $12 \mathrm{~mm}$ long) was placed in front of the detector in order to define the path observed through the flow. The energy window of the analyzer was centered at $27.5 \mathrm{keV}$ to minimize spurious counts due to background radiation and Compton scattering (the latter effect, however, is small for the present energy range).

Absorption measurements (based on roughly 25,000 counts) were made for 30-60 parallel paths through the flow and deconvoluted following Santoro et al. ${ }^{17}$ to yield radial distributions of void fraction. Gomi and Hasegawa ${ }^{18}$ point out that this technique has fundamental uncertainties, depending on whether parallel or normal liquid laminae are assumed; however the narrow absorption paths used during present measurements reduced this effect to less than $5 \%$. The measurements were calibrated using both water cells and the near-injector region of smooth liquid jets at low flow rates. Experimental uncertainties were largely due to finite sampling times and are estimated (95\% confidence) to be less than $5 \%$ for centerline mean liquid volume fractions and proportionaly higher elsewhere.

\section{Laser Doppler Anemometry}

Mean and fluctuating liquid velocities at the injector exit and mean entrainment rates were measured using LDA. The green line $(514.5 \mathrm{~nm})$ of an argon-ion laser $(4 \mathrm{~W}$, Coherent, INNOVA 90-4) was used in a dual-beam, frequency-shifted (40 MHz Bragg cell, TSI model 9180-12) arrangement to eliminate effects of directional bias and ambiguity. The LDA signal was collected using a photomultiplier (TSI model 9160) and processed using a burst counter (TSI model 1990C). All measurements involved low burst densities (one scattering particle in the measuring volume) and high data densities (time between validated signals small in comparison to integral time scales); therefore, the analog output of the processor was time averaged to yield unbiased time averages.

LDA measurements of injector exit conditions were obtained for injection into a water-filled windowed chamber. 
Table 1 Test conditions ${ }^{a}$

\begin{tabular}{|c|c|c|c|c|c|}
\hline $\begin{array}{l}\text { Breakup } \\
\text { regime }\end{array}$ & $\begin{array}{c}\text { Flow rate, } \\
\mathrm{kg} / \mathrm{s}\end{array}$ & $\begin{array}{l}\text { Injector } \\
\text { pressure } \\
\text { drop, kPa }\end{array}$ & $\begin{array}{c}R e_{f} \\
\times 10^{-5}\end{array}$ & $W e_{g}$ & $\begin{array}{l}W e_{f} \\
\times 10^{3}\end{array}$ \\
\hline \multicolumn{6}{|c|}{$9.5 \mathrm{~mm}$ diam, fully developed and slug flows, $O h=12.1 \times 10^{-4}$} \\
\hline $\begin{array}{l}\text { 1st wind-induced } \\
\text { 2nd wind-induced } \\
\text { Atomization }\end{array}$ & $\begin{array}{l}0.39 \\
1.55 \\
3.99\end{array}$ & $\begin{array}{r}80 \\
420 \\
2520\end{array}$ & $\begin{array}{l}0.52 \\
2.07 \\
5.34\end{array}$ & $\begin{array}{r}4.6 \\
72.9 \\
492.8\end{array}$ & $\begin{array}{r}3.9 \\
62.1 \\
411.5\end{array}$ \\
\hline \multicolumn{6}{|c|}{$19.1 \mathrm{~mm}$ diam, fully developed flow, $O h=8.6 \times 10^{-4}$} \\
\hline $\begin{array}{l}\text { 1st wind-induced } \\
\text { 2nd wind-induced } \\
\text { Atomization }\end{array}$ & $\begin{array}{r}1.32 \\
4.50 \\
11.00\end{array}$ & $\begin{array}{r}30 \\
360 \\
2070\end{array}$ & $\begin{array}{l}0.88 \\
3.00 \\
7.32\end{array}$ & $\begin{array}{r}6.6 \\
76.7 \\
458.6\end{array}$ & $\begin{array}{r}5.6 \\
65.4 \\
390.7\end{array}$ \\
\hline
\end{tabular}

a Pressure-atomized water injected vertically downward in still air at $98.8 \mathrm{kPa}$, $298 \pm 2 \mathrm{~K}$, with a constant-area passage 41 diam long for fully developed flow: $R e_{f}=\rho_{f} u_{0} d / \mu_{f}, W e_{i}=\rho_{i} u_{0}^{2} d / \sigma, O h=\mu_{f} /\left(\rho_{f} d \sigma\right)^{1 / 2}$.

The initial beam spacing was $50 \mathrm{~mm}$, sending and receiving optics had $250 \mathrm{~mm}$ focal lengths, and signal detection was 30 deg off-axis in the forward-scattering direction. This yielded a measuring volume having a diameter of $110 \mu \mathrm{m}$ and a length of $220 \mu \mathrm{m}$, which was positioned in a plane 0.1 injector diameters from the injector exit. The local water supply contained adequate natural seeding. Streamwise and radial velocities were measured by orienting the laser beam plane appropriately. Experimental uncertainties $(95 \%$ confidence) were largely governed by finite sampling times and were less than $5 \%$ for mean velocities, $10 \%$ for fluctuating velocities, at the axis, and proportionately higher elsewhere.

The only change in the optical configuration for the entrainment measurements was the use of $600 \mathrm{~mm}$ focal length sending and receiving optics, yielding a measuring volume having a diameter of $260 \mu \mathrm{m}$ and a length of $520 \mu \mathrm{m}$. Mean streamwise and radial entrainment velocities were measured near the edge of the flow, with the entire test cell seeded with condensed bay oil particles (ca. $1 \mu \mathrm{m}$ diameter). These measurements were integrated to provide entrainment rates, with experimental uncertainties ( $95 \%$ confidence) estimated to be less than $25 \%$.

\section{Test Conditions}

Test conditions are summarized in Table 1. Three conditions were examined for each injector, corresponding to first wind-induced, second wind-induced, and atomization breakup. These determinations were based on present observations of the flows. Ranz ${ }^{1}$ suggests $W e_{f}>8$ and $0.4<W e_{g}<13$ for wind-induced breakup and $W e_{f}>8$ and $W e_{g}>13$ for atomization, while Miesse ${ }^{19}$ suggests $W e_{g}>40.3$ for atomization. The results of Table 1 are in rough accord with these criteria, even though present injectors are an order of magnitdue larger than those used by Ranz ${ }^{1}$ and Miesse. ${ }^{19}$ The main difference is that the present second wind-induced conditions are slightly beyond the estimated transitions to atomization; however, in these cases, breakup was relatively close to the injector $(x / d \sim 3)$ and was not far removed from atomization conditions.

\section{Theoretical Methods}

\section{General Description}

The LHF analysis was similar to past work in this laboratory, ${ }^{6,7}$ aside from changes needed to treat the specifics of water injection into air. In addition to the LHF approximation, the major assumptions of the analysis are as follows: steady (in the mean) axisymmetric flow with no swirl, boundary-layer approximation apply, negligible kinetic energy and viscous dissipation of the mean flow, buoyancy affects only the mean flow, and equal exchange coefficients of all species and phases. With the exception of the last, these assumptions are either conditions of the experiments or are justified by successful use in the past. ${ }^{6,7,20}$ The assumption of equal exchange coefficients of both phases is suspect wherever molec- ular transport is important, since molecular transport of finite-sized dispersed-phase elements is small.6,7 However, molecular transport is not very important at the high Reynolds numbers of present flows; therefore, this approximation does not introduce serious limitations in comparison to the general uncertainties of LHF analysis for multiphase jets.

\section{Governing Equations}

Under the present assumptions, all scalar properties are functions of only the mixture fraction (mixture fraction is defined as the fraction of mass at a point that originated from the injector). This allowed use of the conserved-scalar formalism for scalar properties, similar to Lockwood and Naguib, ${ }^{21}$ but based on mass-weighted (Favre) averages following Bilger. ${ }^{22}$

Governing equations are solved for conservation of mass, streamwise mean momentum, mean mixture fraction, turbulence kinetic energy, rate of dissipation of turbulence kinetic energy, and mean-squared mixture fraction fluctuations (see Refs. $6,7,15$, and 20 for the specific formulation and empirical constants). The present approach was successfully calibrated for a variety of constant and variable density singlephase round jets. ${ }^{20}$ The formulation and constants, however, are not very different from those used by Lockwood and Niguib. 21

Initial conditions for the calculations were specified at the injector exit, based on the LDA velocity measurements. It was found that the long injector passages yielded properties at the injector exit which were quivalent to fully developed pipe flow. Rather than interpolate from the measurement plane (at $x / d=0.1), \bar{u}, k$, and $\epsilon$ were taken from Hinze ${ }^{23}$ and Schlicting ${ }^{24}$; while $f=1$ and $g=0$ by definition at the injector exit. (Note that Favre- and time-averaged quantities are identical for the single-phase flow at the injector exit.)

Flow properties were uniform at the exit of the slug-flow injector, except for a narrow layer that could not be resolved using the LDA. In the constant property portion of the flow, $\bar{u}$ was known from the measurements; $\bar{k}$ was computed from measurements of $\bar{u}^{\prime 2}$ and $\bar{v}^{\prime 2}$, assuming $\bar{w}^{\prime 2}=\bar{v}^{\prime 2}$; and $\epsilon$ was approximated as $1.274 \times 10^{-4} \bar{u}^{3} / d$, similar to past work. ${ }^{6,7}$ Properties in the boundary layer along the wall were estimated for a range of $L / d$, bounding reasonable estimates of flow development lengths, assuming clean entry and no vena contracta. These properties were obtained from Schlichting. ${ }^{24}$

\section{State Relationships}

Under present assumptions, scalar properties are functions only of the mixture fraction, called state relationships. ${ }^{6,7}$ State relationships were found by straightforward thermodynamic calculations for adiabatic mixing and equilibrium of various mixtures of injected and ambient fluid. Calculations were completed for the limiting conditions of dry and water-vaporsaturated ambient air. The effect of drop evaporation for dry air, however, was small; therefore, only a water-vaporsaturated environment will be considered here.

When ambient air is fully saturated, there is no tendency for the injected water to evaporate and the flow corresponds to an isothermal mixing process of the gas and liquid, each having constant densities. Thus, variable-density effects are due only to mixing of the phases under the LHF approximation. The state relationships for mixture density and liquid volume fraction are

$$
\begin{gathered}
\rho=\left[f / \rho_{f}+(1-f) / \rho_{g}\right]^{-1} \\
\alpha_{f}=\left[1+\left(\rho_{f} / \rho_{g}\right)(1-f) / f\right]^{-1}
\end{gathered}
$$

The state relationships were then used to find time- and Favreaveraged scalar properties, assuming a clipped Gaussian Favre-averaged probability density function for mixture fraction, similar to earlier work. ${ }^{6,7,22}$ 


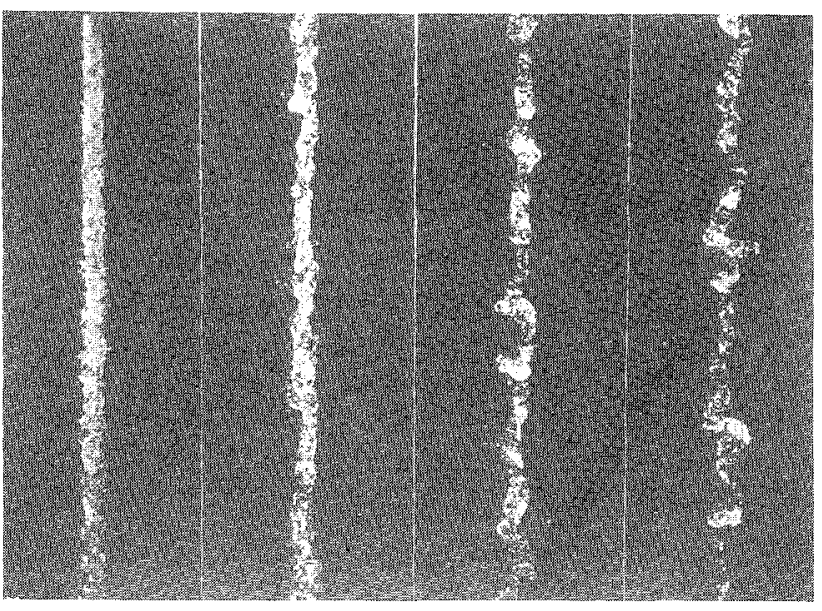

a) First wind-induced breakup

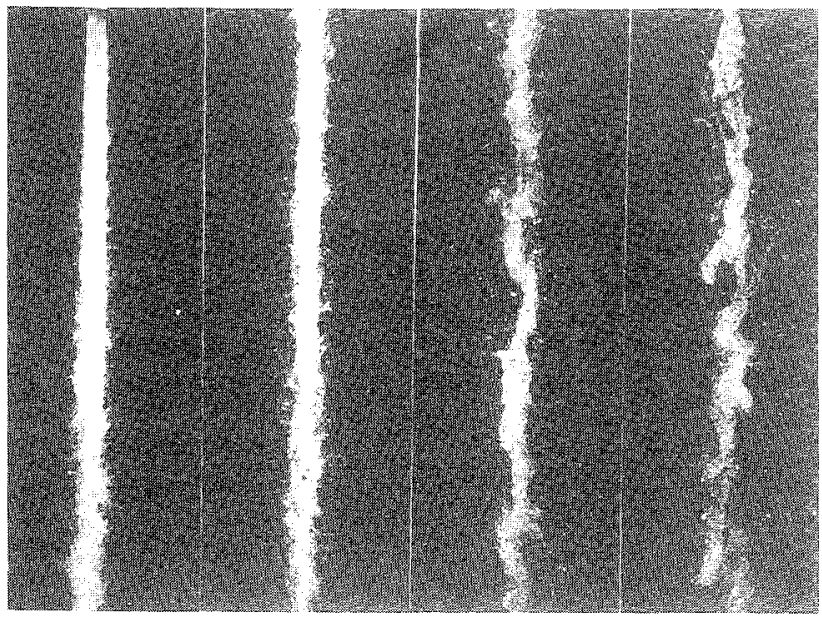

b) Second wind-induced breakup
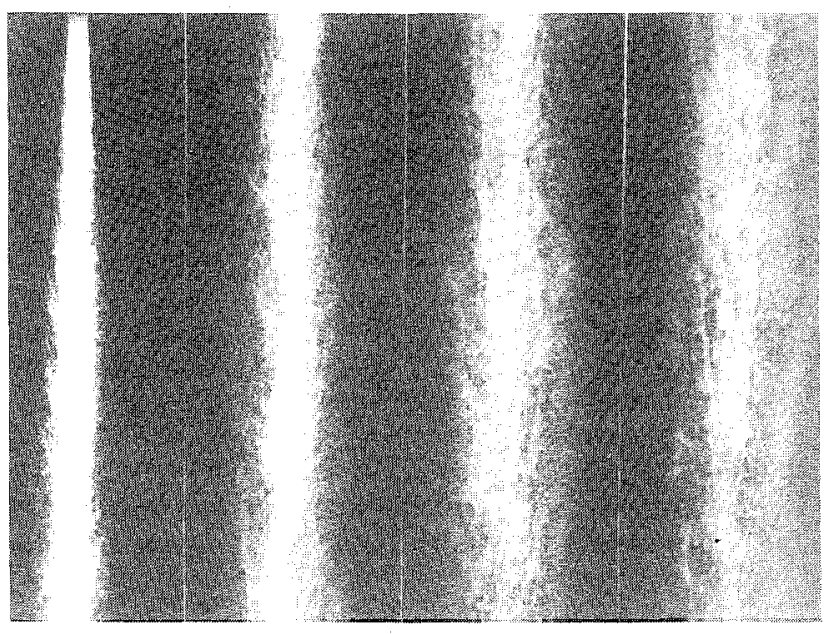

c) Atomization breakup

Fig. 1 Flash photographs for the $9.5 \mathrm{~mm}$ diam injector and fully developed flow.

\section{Numerical Solution}

The governing equations were solved using GENMIX. ${ }^{25}$ The large density variations of the flows caused problems of computational stability and numerical accuracy, requiring much finer grids than are usually needed for single-phase flows. Present slug and fully developed flow computations used 360 and 720 cross-stream grid nodes with streamwise step sizes limited to 0.30 and $0.15 \%$ of current flow width, respectively. Doubling the number of grid nodes in both the crossstream and streamwise directions changed predictions by less than $1 \%$.

\section{Results and Discussion}

\section{Flow Visualization}

Typical flash photographs for fully developed flow in the first wind-induced, second wind-induced, and atomization breakup regimes are illustrated in Fig. 1. These results were obtained using the $9.5 \mathrm{~mm}$ diam injector; however, findings for the $19.1 \mathrm{~mm}$ diam injector were similar. Four pictures are shown for each test condition: near the injector exit and centered at $x / d=50,100$, and 150 . The lowest position appearing in the photographs is nearly $2 \mathrm{~m}$ from the injector exit.

For first wind-induced breakup, the liquid surface exhibits fine-grained roughness near the injector exit, which becomes smoother, with large-scale irregularities appearing, far from the injector. This suggests shifts in the turbulence spectra of both phases. Liquid-phase turbulence properties near the injector exit are governed by the injector passage. Once the flow leaves the passage, however, the mean liquid velocities become more uniform, since the gas cannot retard the surface velocity as effectively as the wall of the injector passage. This reduces turbulence production in the liquid, causing the turbulence to decay with the small-scale, high wave-number end of the spectrum disappearing first. The developing flow in the gas phase also favors the smallest scales near the injector exit. However, gas-phase turbulence probably does not have a strong influence on liquid surface properties at atmospheric pressure, since the gas density is small in comparison to the liquid. This was confirmed since the liquid surface exhibited little finegrained roughness near the injector exit for slug flow, which had low initial turbulence intensities. ${ }^{15}$ Thus, for present conditions, liquid-phase turbulence properties at the injector exit dominate roughness of the liquid surface and probably influence the drop size distributions once breakup occurs as well. For first wind-induced breakup, however, the large-scale irregularities (kinks) of the liquid column eventually cause breakup into the large liquid elements that are characteristics of this breakup regime.

In the second wind-induced breakup regime, the liquidphase turbulence again produces small-scale surface roughness. However, these disturbances grow and are sheared from the surface, producing a cloud of drops surrounding the liquid core. The point where breakup begins is roughly three injector diameters from the injector exit for the condition illustrated in Fig. 1. The character of the surface roughness influences breakup, e.g., slug-flow conditions at the same flow rate, where the liquid surface was relatively smooth, caused the point of breakup to move downstream to 25-30 injector diameters from the exit. ${ }^{15}$ Present criteria for breakup regime transitions do not account for effects of flow development at the injector exit, which clearly affect the onset of atomization-extending the criteria to account for these effects is clearly needed. In spite of breakup at the surface, however, an all-liquid core can clearly be seen in the flow. This liquid core eventually breaks up far from the injector, probably yielding some rather large drops, similar to first wind-induced breakup.

As noted earlier, increasing injector velocities in the second wind-induced breakup regime cause the point of breakup of the surface of the liquid column to move toward the injector exit. When breakup reaches the exit, the atomization breakup regime is entered and is observed for all higher injector flow rates. the atomization condition illustrated in Fig. 1 is well within the atomization regime. The wispy appearance of the drop-containing region near the edge of the flow, similar to a single-phase flow containing tracer particles, suggests that the drops near the exit are small. Clear areas of drop intermittency, also similar to single-phase turbulent shear layers, penetrate the drop-containing region. The extent of penetration is relatively small near the injector exit, suggesting the presence of an all-liquid core similar to the other breakup regimes illustrated in Fig. 1. The depth of penetration of the drop-free regions increases with increasing distance from the injector, but drop intermittency is not seen at the axis until $x / d \sim 150-200$. 


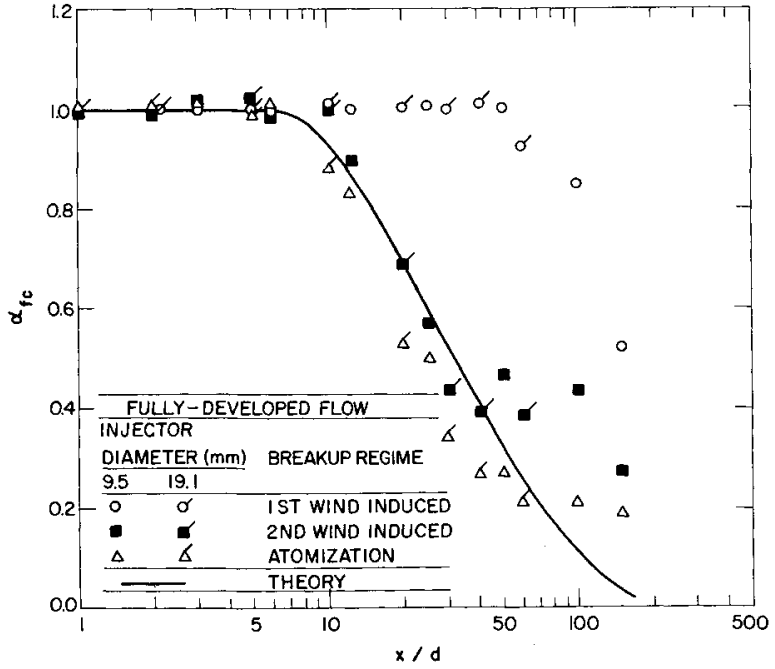

Fig. 2 Time-averaged liquid volume fractions along the axis for fully developed flow.

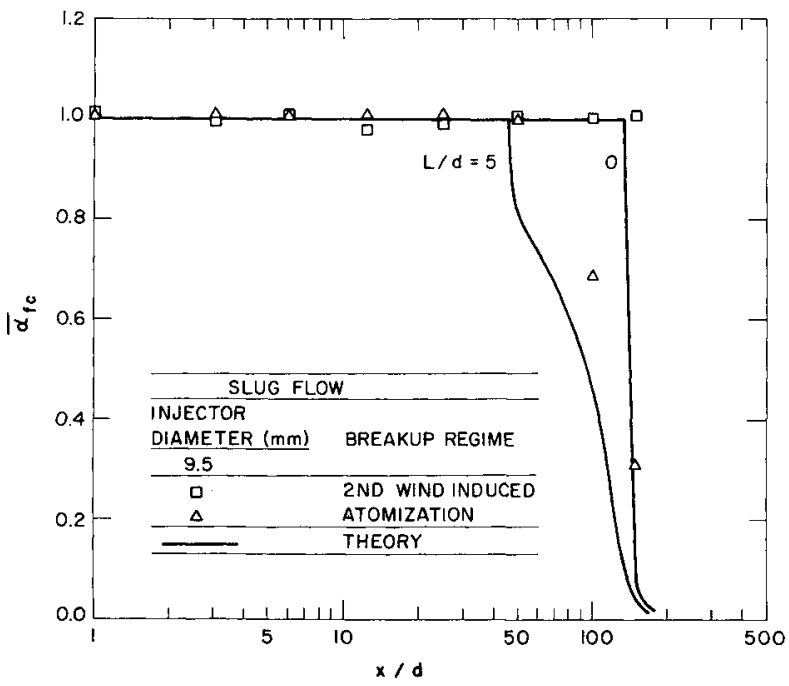

Fig. 3 Time-averaged liquid volume fractions along the axis for slug flow.

The spray had a more opaque, milky appearance for slug flow than for fully developed flow and atomization breakup, suggesting higher concentrations of smaller drops in the dropcontaining shear layer. ${ }^{15}$ However, the rate of spread of the shear layer (indicated by the extent of the region where drops scattered significant light on the photographs) was significantly smaller and the appearance of drop intermittancy along the axis was delayed for slug flow. This implies a longer allliquid core for slug flow than for fully developed flow at the same injector flow rate. This behavior is confirmed by the liquid volume fraction measurements to be considered next.

\section{Mean Liquid Volume Fractions}

Measured and predicted time-averaged mean liquid volume fractions along the axis of the fully developed and slug flow sprays are plotted as a function of $x / d$ in Figs. 2 and 3. Fully developed flows included 9.5 and $19.1 \mathrm{~mm}$ diam injectors and all three breakup regimes. Slug flows were limited to the 9.5 $\mathrm{mm}$ diam injector and the second wind-induced and atomization breakup regimes.

For fully developed flow (Fig. 2), the near-injector region $(x / d<10)$ exhibits liquid volume fractions near unity. For $x / d>10$, however, liquid volume fractions decrease rapidly in the second wind-induced and atomization breakup regimes, reaching values of $0.2-0.3$ at $x / d=150$. For $x / d<40$, the measurements in the second wind-induced and atomization
Table $2 \alpha_{f}$ vs $f$ at $846: 1$ density ratio

\begin{tabular}{rlllll}
\hline$f$ & 1.0000 & 0.9999 & 0.9990 & 0.9900 & 0.9000 \\
$\alpha_{f}$ & 1.000 & 0.922 & 0.541 & 0.105 & 0.011 \\
\hline
\end{tabular}

regimes are similar when the streamwise distance is normalized by the injector diameter. This behavior suggests a turbulent mixing-controlled process in the near-injector region, which might be amenable to analysis using the LHF approximation.

In contrast, mixing is much slower for the first windinduced breakup regime than the other breakup regimes illustrated in Fig. 2 for fully developed flow. In this case, liquid volume fractions remain near unity for $x / d<50$, followed by a region of rapid reduction of mean liquid volume fractions along the axis. In the region where the mean liquid volume fraction declines, however, apparent mixing is due only to lateral deflection of the liquid column (the formation of kinks and lateral flapping of the column as a whole), rather than the more complete mixing caused by the formation of drops; see Fig. 1. However, flow properties for first wind-induced breakup are seen to also scale with injector diameter.

The results illustrated in Fig. 2 superficially suggest a relatively short all-liquid core near the injector exit, particularly for second wind-induced and atomization breakup. This is not actually the case. Due to the large water/air density ratio of the flow, liquid volume fraction is a very sensitive function of mixture fraction. This can be seen in Table 2, which lists $\alpha_{f}$ as a function of $f$, under the LHF approximation, for the present density ratio (846:1). It can be seen that mixture fraction decreases only $1 \%$ while the liquid volume fraction decreases by a factor of 10 , from 1 to 0.1 . Thus, all the results illustrated in Fig. 2 represent mixture fractions greater than 0.99. (Note that with liquid velocities greater than gas velocities, the reduction of $f$ would be even smaller if the LHF approximation was not valid.) Thus, as expected, mixing is much slower for the present liquid jets than for single-phase flows, ${ }^{12}$ where $\tilde{f}_{c} \sim 0.03$ at $x / d=150$. Long liquid cores are present for all the flows considered here, when viewed in terms of mixture fraction.

LHF predictions are essentially independent of Reynolds numbers for the high Reynolds numbers of present tests; therefore, the single predictions appearing in Fig. 2 represents all test conditions for fully developed flow. Measurements for second wind-induced breakup are in good agreement with this prediction for $x / d<40$, while similar agreement is observed for atomization breakup when $x / d<100$. At greater distances, measured liquid volume fractions are greater than predictions, suggesting increased effects of relative velocities between the phases. It is plausible that results for second wind-induced breakup depart from predictions sooner than atomization breakup, since drop sizes are larger for second wind-induced breakup, providing greater potential for significant relative velocities between the phases. Failure of the LHF approximation also occurs slightly sooner (in terms of $x / d$ ) for the smaller injector, since smaller passage diameters yield higher flow decelaration rates ${ }^{6,7}$ (which scales as $u_{0} / d$ ) that cause higher relative velocities in the flow. Such higher relative velocities become significant in comparison to local flow velocities nearer to the injector, reducing the regions where the LHF approximation is adequate. These effects eventually become important for all the flows considered in Fig. 2, causing LHF predictions to fail far from the injector as the dilutespray region is approached. This observation is consistent with deficiencies reported in the past for LHF analysis of dilute sprays. ${ }^{6,7}$

Comparing results for atomization breakup for fully developed and slug flows at the injector exit (cf. Figs. 2 and 3) shows that effects of flow development at the injector exit are nearly as dramatic as effects of the breakup regime. The physical reason for this behavior is that the liquid density is large in comparison to the gas; therefore, fully developed flow carries significant levels of turbulence energy into the flow, enhancing 


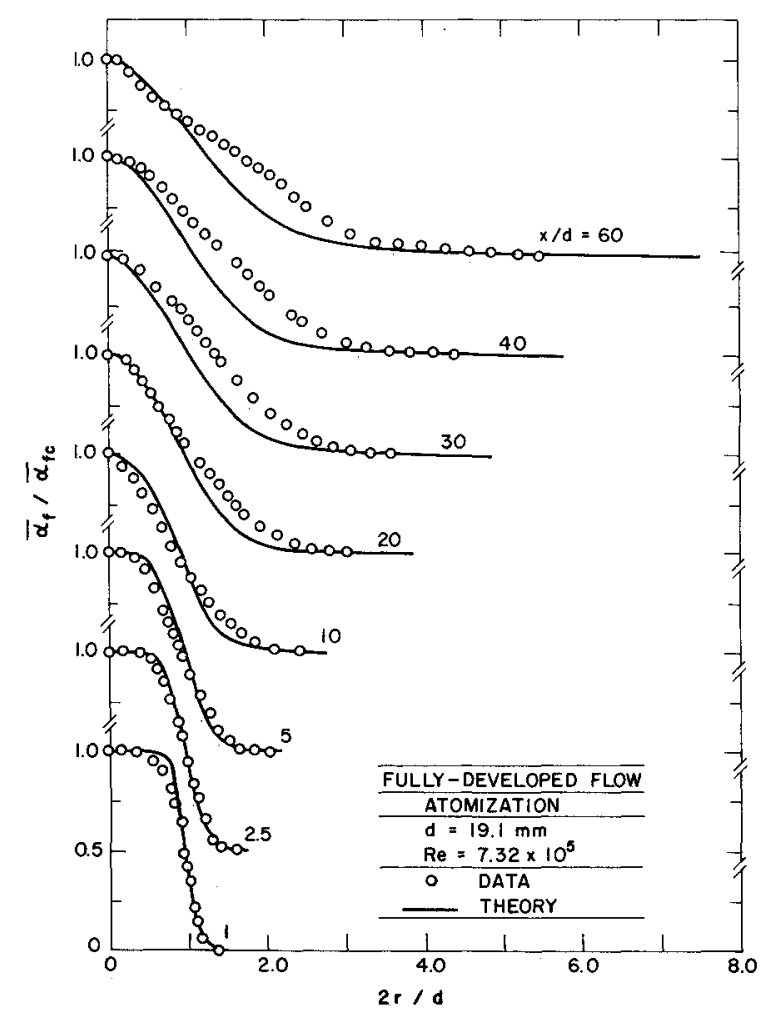

Fig. 4 Radial profiles of time-averaged liquid volume fractions for fully developed flow and atomizaton breakup $(19.1 \mathrm{~mm}$ diam injector).

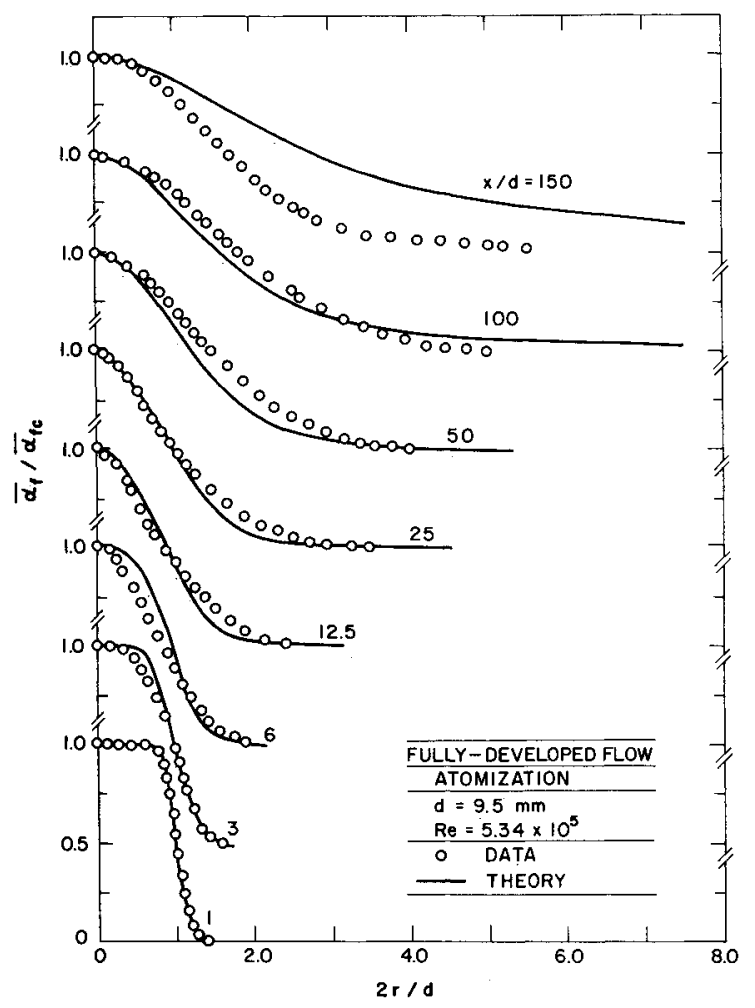

Fig. 5 Radial profiles of time-averaged liquid volume fractions for fully developed flow and atomization breakup $(9.5 \mathrm{~mm}$ diam injector).

mixing in the region where mixture fractions are high. As noted earlier, this liquid-phase turbulence also creates instabilities in the liquid surface that would provide more rapid breakup of liquid drops-and enhance mixing as well.

These effects cause slower initial rates of flow development for slug flow than for fully developed flow, e.g., liquid volume fractions for slug flow remain near unity for $x / d<50$

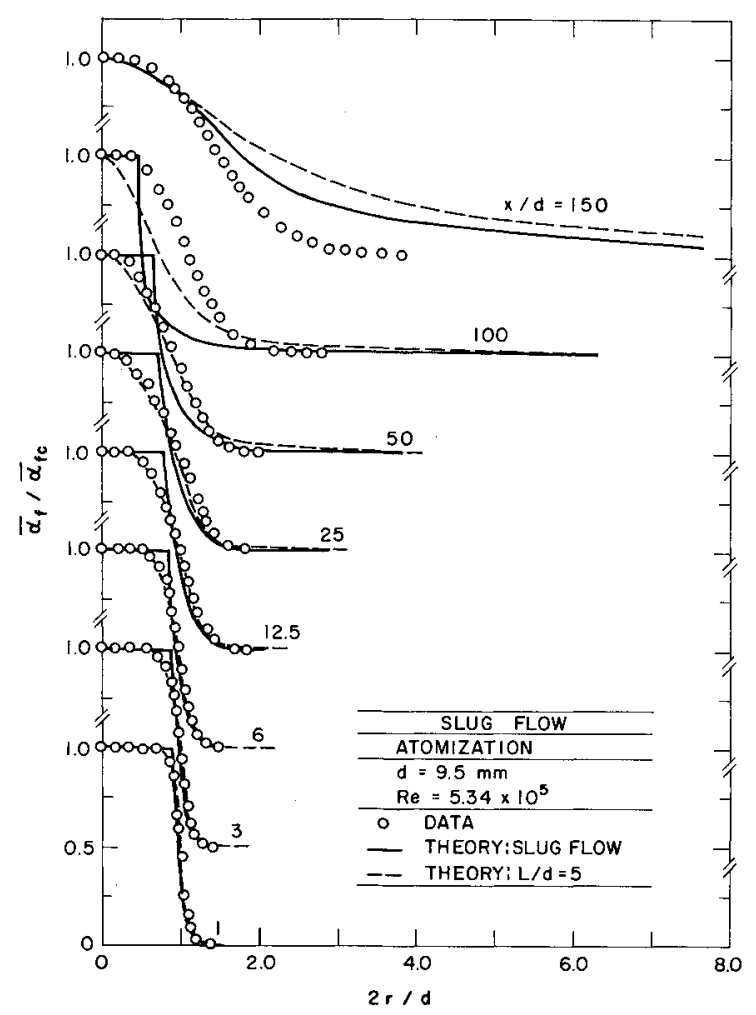

Fig. 6 Radial profiles of time-averaged liquid volume fractions for slug flow and atomization breakup (9.5 $\mathrm{mm}$ diam injector).

as opposed to $x / d<10$ for fully developed flow. However, later development of the flow is rapid, resulting in mean liquid volume fractions near 0.3 at $x / d=150$. This implies a relatively long all-liquid core, particularly when viewed in terms of mixture fraction, as noted earlier.

Predictions illustrated in Fig. 3 are relatively independent of Reynolds number, but are strongly influenced by the degree of flow development at the injector exit (which is represented by the passage $L / d$ ). It is encouraging that computations for $L / d=0$ and 5 , which are reasonable limits for the test injector, tend to bound the measurements for atomization breakup. Comparing the results for atomization breakup in Figs. 2 and 3 shows that LHF predictions properly represent the strong effect of the degree of flow development at the injector exit on the subsequent mixing of the spray in the near-injector region. The effect of flow development is probably a contributing factor in controversies concerning the properties of the all-liquid core based on measurmenets from short $L / d$ injectors. ${ }^{3-5}$

Radial profiles of time-averaged liquid volume fractions for atomization breakup are illustrated in Figs. 4-6. Measurements and predictions are plotted as a function of radial distance, normalized by the injector diameter, at various distances from the injector exit. Results for fully developed flow for the 19.1 and $\mathbf{9 . 5} \mathrm{mm}$ diam injectors are plotted in Figs. 4 and 5 and similar results for slug flow in Fig. 6.

The apparent flow widths seen in Figs. 4-6 are unusually narrow in comparison to single-phase jets. For example, flow widths are on the order of $2 r / d=4-6$ for $x / d=100-150$, which correspond to $r / x$ on the order of 0.02 . In comparison, widths of single-phase jets are nearly an order-of-magnitude larger, ca. $r / x=0.15 .26$ The sensitivity of the liquid volume fraction to mixture fraction, mentioned earlier, is the main cause of this behavior; e.g., based on Eq. (2), profiles of mixture fraction are much wider than liquid volume fraction. The extended all-liquid core for present flows also contributes to this behavior, since liquid that has not become atomized cannot mix very effectively.

For fully developed flow, the comparison between predicted and measured radial profiles of mean liquid volume fractions seen in Figs. 4 and 5 can be anticipated from the results along 
the axis illustrated in Fig. 2. Conditions where $\bar{\alpha}_{f c}$ is predicted well, e.g., $x / d<100$, also result in reasonably good predictions of radial profiles. All predictions far from the injector, however, overestimate the width of the flow. This agrees with past evaluations of LHF analysis in the dilute portions of sprays, which showed that the method invariably overestimates measured rates of flow development. ${ }^{6,7,12-14}$ This occurs since relative velocities become significant whenever flow velocities are low, which roughly corresponds to the dilutespray region.

Results for slug flow are illustrated in Fig. 6. In this case, predictions are plotted for the limits of $L / d=0$ and 5 . Near the injector, there is a reasonably wide zone where liquid volume fractions are near unity. Predictions suggest a sharp transition between this region and the shear layer, while measurements show a more gradual transition between these regions. This discrepancy is partly due to gradient-broadening errors of the measurements, where the finite diameter of the paths of the absorption measurements cannot resolve the rapid radial variations of the mean liquid volume fractions. On the other hand, the discontinuity of the predictions is due to approximations made to match calculations in the shear layer with the all-liquid core and it probably not observed in nature.

The discrepancies between predictions and measurements are largest at $x / d=100$ for slug flow. Both predictions underestimate rather than overestimate the flow width which is the expected behavior of the LHF approximation. This behavior is caused by the discrepancies between measured $\bar{\alpha}_{f c}$ and predictions for the limits of $L / d=0$ and 5 near $x / d=100$ (see Fig. 3 ). Thus, the poor agreement at $x / d=100$ is due largely to the $\bar{\alpha}_{f} / \bar{\alpha}_{f c}$ normalization used in Fig. 6.

For slug flow and $x / d=150$, corresponding to low values for $\bar{\alpha}_{f c}$, the predictions for $L / d=0$ and 5 overestimate the development of the jet, similar to fully developed flow. However, the slug flow predictions do not depart as much from the measurements as for the fully developed flow at this position (see Fig. 5). Two reasons can be suggested for this behavior. First of all, slug flow develops more slowly than fully developed flow, providing a more extended shear layer region with lower rates of decelaration and, thus, tending to favor the LHF approximation. Second, as noted earlier, it appears that smaller drops are generated for slug flow than for fully developed flow, which also favors the LHF approximation. Based on the Taylor ${ }^{27}$ breakup criterion, discussed by Reitz and Bracco, ${ }^{2}$ the production of smaller drops for slug flow than fully developed flow is plausible because of the higher velocity gradients near the liquid surface. However, direct measurements of drop size and velocity properties in the near-injector region are needed to provide more information concerning these conjectures.

\section{Entrainment Rates}

The entrainment rate of a jet is proportional to the rate of increase of the jet mass flow rate with distance from the injector and is a good measure of the turbulent mixing properties of the flow. ${ }^{28}$ Measured and predicted entrainment rates for the present flows are illustrated in Fig. 7. Normalized entrainment rates are plotted as a function of distance from the injector, considering all three breakup regimes for fully developed flow and second wind-induced and atomization breakup for slug flow. As noted earlier, present predictions did not vary significantly with Reynolds number; therefore, the predictions illustrated in Fig. 7 are representative of the Reynolds number range of the experiments. As before, predictions at the limits of $L / d=0$ and 5 were made for slug flow. However, the results were nearly the same; therefore, only a single prediction line appears in Fig. 7. The entrainment rate correlation of Ricou and Spalding ${ }^{28}$ is also plotted in Fig. 7: this correlation was developed for fully developed flow $(x / d>20)$ of variabledensity turbulent gas jets.

Measured normalized entrainment rates increase with increasing distance from the injector. The entrainment rates for

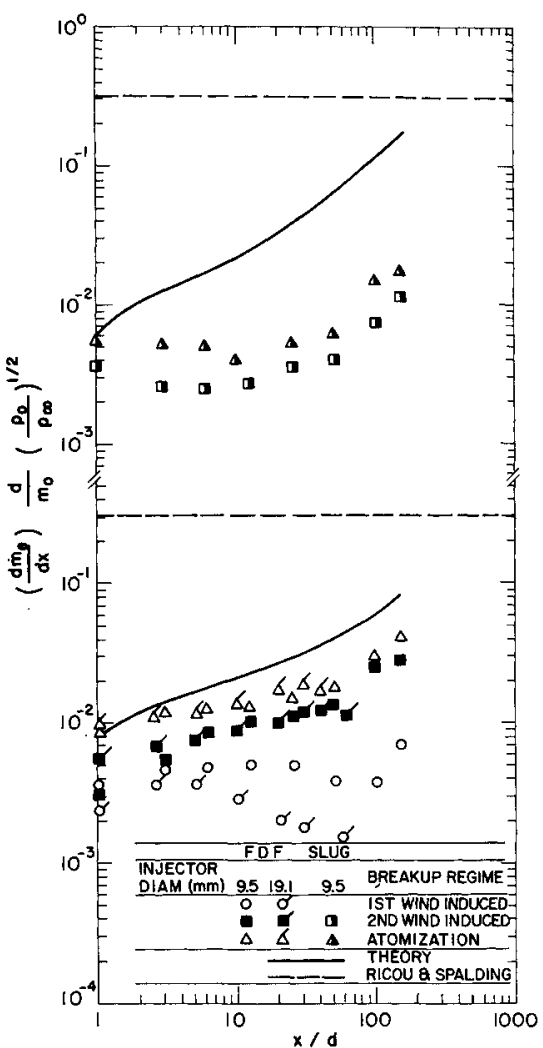

Fig. 7 Entrainment rates as a function of distance from the injector.

first wind-induced breakup are substantially lower than for second wind-induced breakup and atomization, which differ only slightly from each other. This is plausible, since the liquid column for first wind-induced breakup does not shatter into drops for the present test range; therefore, there is little surface area available to promote momentum exchange and turbulent mixing.

In general, predictions substantially overestimate measurements for all the results illustrated in Fig. 7. Furthermore, the correlation of Ricou and Spalding ${ }^{28}$ yields much larger estimates of entrainment than present measurements and predictions. This is due to effects of both flow development and finite relative velocities. Differences between the correlation of Ref. 28 and present LHF predictions are a measure of effects of flow development. This is caused by differences between present velocity and density distributions in the developing flow near the injector and velocity profiles for fully developed turbulent gas jets, which have rather modest density variations in the fully developed region of flow. In the terminology of integral theories, this is a shape-factor effect, resulting from dissimilar velocity and scalar property profiles. Similarity in this sense implies invariance of radial profiles when normalized by centerline quantities and plotted as a function of $r / x$ : the results illustrated in Figs. 4-6 show that this requirement is not satisfied for the present flows-even for atomization breakup.

Present predictions allow for effects of flow development, but still significantly overestimate measured rates of entrainment. The discrepancies are smallest for full developed flow and atomization breakup, but even this prediction is not very satisfactory. This behavior follows since entrainment rates are strongly influenced by flow properties near the edge of the flow, where velocities are low and the effects of relative velocities become important. Stated differently, the periphery of the flow, which directly affects entrainment properties, is a dilute-spray region where LHF analysis invariably overestimates rates of flow development, which is represented by properties like the entrainment rate. Similar deficiencies are less apparent for liquid volume fractions, since present measurements emphasize regions having high mixture fractions. Flow 
velocities area also high in these regions, tending to reduce errors due to finite relative velocities.

\section{Sensitivity Study}

The sensitivity of present calculations was examined similar to past evaluations of spray analysis. ${ }^{13,14}$ Predictions were most sensitive to uncertainties in the initial values of $k$ and $\epsilon .{ }^{15}$ However, these parameters were reasonably well known for fully developed injector flows, while affects of turbulence properties in the core of the flow were not very significant for slug flow. Thus, uncertainties in predictions, aside from wellrecognized limitations of $k$ - $\epsilon$ turbulence models for boundarylayer flows, are generally within experimental uncertainties.

A final point should be mentioned with respect to the turbulence model. If the present flows formally satisfied the LHF approximation, they would represent a variable density jet with a density ratio of ca. 1000:1. This is roughly two orders of magnitude greater than the variable-density single-phase flows used to calibrate the present Favre-averaged turbulence model. ${ }^{20}$ Thus, whether these methods can accurately handle the present large density ratios if the LHF assumption was formally satisfied is unknown and deficiencies here could have contributed to discrepancies between predictions and measurements. Based on present findings, however, limitations of the LHF approximation due to finite relative velocities in lowmean-velocity regions of the flow (the dilute-spray region) appear to be a more obvious source of errors in the predictions.

\section{Conclusions}

Major conclusions of the study are as follows:

1) Pressure-atomized sprays are unusually sensitive to the degree of flow development and turbulence levels at the injector exit. Fully developed turbulent flows cause much faster rates of flow development and shorter all-liquid cores than slug flows having low initial turbulence intensities.

2) Locally homogeneous flow analysis was reasonably successful for atomization breakup in the dense-spray, nearinjector region where mean liquid volume fractions are relatively high $\left(\bar{\alpha}_{f}>0.2\right)$. In particular, present methods provided good estimates of the striking effects of flow development at the injector exit, in spite of the large density variations (ca. 1000:1) of the flows.

3) Similar to past findings in this laboratory, ${ }^{10-14}$ the LHF approximation was less effective for properties of the dilutespray region near the periphery of the flow and far downstream from the injector. This deficiency causes flow entrainment rates to be overestimted in the region observed $(x / d<150)$

4) Properties of the all-liquid core near the injector exit are influenced by the breakup regime and the state of flow development at the injector exit. The last effect is probably a factor causing differences in all-liquid core lengths reported by various workers. ${ }^{3-5}$

Present conclusions are based on large-scale sprays $(9.5$ and $19.1 \mathrm{~mm}$ injector diameters) that have much lower deceleration rates than practical injectors-favoring use of the LHF approximation. Present results are also limited to water injected into air at normal temperature and pressure; other liquids and ambient gases will modify drop-size distributions and probably the effectiveness of the LHF approximation as well. Additional study of this important and fundamental multiphase flow is clearly needed.

\section{Acknowledgments}

This research was sponsored by the Air Force Office of Scientific Research, Air Force Systems Command, USAF, under Grant AFOSR-85-0244. The U.S. Government is authorized to reproduce and distribute reprints for Governmental purposes notwithstanding any copyright notation thereon.

\section{References}

${ }^{1}$ Ranz, W. E., "Some Experiments on Orifice Sprays," Canadian Journal of Chemical Engineering, Vol. 36, Aug. 1958, pp. 175-181.
${ }^{2}$ Reitz, R. D. and Bracco, F. V., "Mechanism of Atomization of a Liquid Jet," Physics of Fluids, Vol. 25, Oct. 1982, pp. 1730-1742.

${ }^{3}$ Cheroudi, B., Onuma, Y., Chen, S.-H., and Bracco, F. V., "On the Intact Core of Full-Cone Sprays," Society of Automotive Engineers, Warrendale, PA, SAE Paper 850126, 1985.

${ }^{4}$ Hiroyasu, H., Shimizu, M., and Arai, M., "The Breakup of a High Speed Jet in a High Pressure Gaseous Environment," Univ. of Wisconsin, Madison, ICLASS-82, 1982.

${ }^{5}$ Phinney, R. E., "The Breakup of a Turbulent Liquid Jet in a Gaseous Atmosphere," Journal of Fluid Mechanics, Vol. 60, Oct. 1973, pp. 689-701.

${ }^{6}$ Faeth, G. M., "Evaporation and Combustion in Sprays," Progress in Energy and Combustion Science, Vol. 9, 1983, pp. 1-76.

${ }^{7}$ Faeth, G. M., "Mixing, Transport and Combustion in Sprays," Progress in Energy and Combustion Science, Vol. 13, 1987, pp. 293-345.

${ }^{8}$ Wu, K.-J., Su, C.-C., Steinberger, R. L., Santavicca, D. A., and Bracco, F. V., "Measurements of the Spray Angle of Atomizing Jets," Journal of Fluids Engineering, Vol. 105, Dec. 1983, pp. 406-415.

${ }^{9}$ Wu, K.-J., Coghe, A., Santavicca, D. A., and Bracco, F. V., "LDV Measurements of Drop Velocity in Diesel-Type Sprays," AIAA Journal, Vol. 22, Sept. 1984, pp. 1263-1270.

${ }^{10}$ Mao, C.-P., Wakamatsu, Y., and Faeth, G. M., "A Simplified Model of High Pressure Spray Combustion," Eighteenth Symposium (International) on Combustion, The Combustion Inst., Pittsburgh, PA, 1981, pp. 337-347.

${ }^{11}$ Mao, C.-P., Szekely, G. A., Jr., and Faeth, G. M., "Evaluation of a Locally Homogenous Flow Model of Spray Combustion," Journal of Energy, Vol. 4, March-April 1980, pp. 78-87.

${ }^{12}$ Shearer, A. J., Tamura, H., and Faeth, G. M., "Evaluation of a Locally Homogenous Flow Model of Spray Evaporation," Journal of Energy, Vol. 3, Sept.-Oct. 1979, pp. 271-278.

${ }^{13}$ Solomon, A. S. P., Shuen, J.-S., Zhang, Q.-F., and Faeth, G. M., "Structure of Nonevaporating Sprays: I. Near-Injector Conditions and Mean Properties," AIAA Journal, Vol. 23, Oct. 1985, pp. 1548-1555.

${ }^{14}$ Solomon, A. S. P., Shuen, J.-S., Zhang, Q.-F., and Faeth, G. M., "Structure of Nonevaporating Sprays: II. Drop and Turbulence Properties," AIAA Journal, Vol. 23, Nov. 1985, pp. 1724-1730.

${ }^{15}$ Ruff, G. A. and Faeth, G. M., "Dense-Spray Structure and Phenomena: Part II. Pressure-Atomized Sprays," Univ. of Michigan, Ann Arbor, Interim Rept. under Grant AFOSR-85-0244, Sept. 1987.

${ }^{16}$ Smith, R. H. and Wang, C.-T., "Contracting Cones Giving Uniform Throat Speeds," Journal of the Aeronautical Sciences, Vol. 11, Oct. 1944, pp. 356-360.

${ }^{17}$ Santoro, R. J., Semerjian, J. H., Emmerman, P. J., and Goulard, R., "Optical Tomography for Flow Field Diagnostics," International Journal of Heat and Mass Transfer, Vol. 24, July 1981, pp. 1139-1150.

${ }^{18} \mathrm{Gomi}, \mathrm{H}$. and Hasegawa, K. I., "Measurements of the Liquid Phase Mass in Gas-Liquid Sprays by X-ray Attenuation," International Journal of Multiphase Flow, Vol. 10, Dec. 1984, pp. 653-662.

${ }^{19}$ Miesse, C. C., "Correlation of Experimental Data on Disintegration of Liquid Jets," Industrial Engineering Chemistry, Vol. 47, Sept. 1955 , pp. 1690-1697.

${ }^{20}$ Jeng, S.-M. and Faeth, G. M., "Species Concentrations and Turbulence Properties in Buoyant Methane Diffusion Flames," Journal of Heat Transfer, Vol. 106, Aug. 1985, pp. 721-727.

${ }^{21}$ Lockwood, F. C. and Naguib, A. S., "The Prediction of Fluctuations in the Properties of Free, Round-Jet Turbulent Diffusion Flames," Combustion and Flame, Vol. 24, Feb. 1975, pp. 109-124.

${ }^{22}$ Bilger, R. W., "Turbulent Jet Diffusion Flames," Progress in Energy and Combustion Science, Vol. 1, 1976, pp. 87-109.

${ }^{23}$ Hinze, J. O., Turbulence, 2nd ed. McGraw-Hill, New York, 1975, pp. 427, 724-734.

${ }^{24}$ Schlicting, H., Boundary Layer Theory, 7th ed., McGraw-Hill, New York, 1979, p. 599.

${ }^{25}$ Spalding D. B., GENMIX: A General Computer Program for Two-Dimensional Parabolic Phenomena, Pergammon, Oxford, England, 1977.

${ }^{26}$ Wygnanski, I. and Fiedler, H. E., "Some Measurements in the Self-Preserving Jet," Journal of Fluid Mechanics, Vol. 38, Sept. 1969 , pp. 577-612.

${ }^{27}$ Taylor, G. I., "Generation of Ripples by Wind Blowing Over a Viscous Fluid," The Scientific Papers of Sir Geoffrey Ingram Taylor, Vol. III, edited by G. K. Batchelor, Cambridge Univ. Press, Cambridge, England, 1963, pp. 244-254.

${ }^{28}$ Ricou, F. P. and Spalding, D. B., "Measurements of Entrainment by Axisymmetrical Turbulent Jets," Journal of Fluid Mechanics, Vol. 11, Aug. 1961, pp. 21-32. 